\title{
Appraisal of donor steatosis in liver transplantation: a survey of current practice in Australia and New Zealand
}

This article was published in the following Dove Press journal:

Transplant Research and Risk Management

13 December 2012

Number of times this article has been viewed

\author{
Anna J Dare' \\ Anthony RJ Phillips ${ }^{1-3}$ \\ Michael Chu' \\ Anthony JR Hickey ${ }^{2}$ \\ Adam SJR Bartlett ${ }^{1-3}$ \\ 'Department of Surgery, ${ }^{2}$ Maurice \\ Wilkins Centre for Biodiscovery, \\ University of Auckland, Auckland, \\ New Zealand; ${ }^{3} \mathrm{New}$ Zealand Liver \\ Transplant Unit, Auckland City \\ Hospital, Auckland, New Zealand
}

\begin{abstract}
Background: Hepatic steatosis is increasingly encountered among organ donors. Currently, there is no consensus guideline as to the type or degree of donor steatosis considered acceptable for liver transplantation (LT), and little is known about local practices in this area. The aim of this survey was to evaluate current clinical practices amongst liver transplant surgeons in Australia and New Zealand (ANZ) in the evaluation and use of steatotic donor livers in LT.
\end{abstract}

Methods: An anonymous online twelve-question survey was emailed to all practicing LT surgeons in ANZ ( $\mathrm{n}=23)$ in January 2010.

Results: The response rate was $83 \%$. Estimated prevalence of steatosis in donor livers was between $40 \%$ and $60 \%$. In determining suitability for LT, $90 \%$ of respondents reported rejecting organs with "severe" steatosis based on visual and palpation grounds alone. A total of $68 \%$ sought further histological assessment if the donor liver looked bad and there were risk factors for steatosis. The majority of respondents performed only one biopsy of the liver (79\%), using hematoxylin and eosin staining for fat assessment (53\%). There was wide variation in the upper limit of steatosis considered to be acceptable for LT ( $40 \%-80 \%$ steatosis). A total of $21 \%$ of respondents still considered microvesicular steatosis a risk factor for primary graft nonfunction.

Conclusion: This survey highlights the significant variation in the appraisal and use of steatotic grafts by LT surgeons in ANZ. Accurate evaluation and judicious use of mild and moderately steatotic grafts is required if we are to utilize the available donor pool best.

Keywords: liver transplantation, steatosis, fatty liver, organ donors

\section{Introduction}

The twenty-first century has seen an exponential rise in the prevalence of obesity, diabetes mellitus, and metabolic syndrome. ${ }^{1}$ The hepatic consequence of these states is the development of nonalcoholic fatty liver disease, with deposition of triglycerides in the liver. Consequently, hepatic steatosis is increasingly seen in patients coming forward for surgery, and in particular organ donation. ${ }^{2}$ In liver transplantation, severe steatosis of the donor liver is a risk factor for primary nonfunction $(\mathrm{PNF})^{3}$ and mild $(<30 \%)$ and moderate $(30 \%-60 \%)$ steatosis has been associated with risk of inferior graft function. ${ }^{4-6}$ For this reason, donor livers with overt hepatic steatosis have been avoided by most centers. However, the increased demand for donor organs over the last decade has forced centers to use marginal or extended criteria donors in LT, including those with evidence of hepatic steatosis. ${ }^{7,8}$ A number of single-center studies have demonstrated acceptable outcomes when using mild and moderately steatotic organs, ${ }^{9-11}$ particularly when carefully matched to appropriate recipients, ${ }^{12}$ though the area is controversial.
Correspondence: Adam Bartlett New Zealand Liver Transplant Unit, Level I5, Support Building, Auckland City Hospital, 2 Park Road, Grafton, Auckland 1023, New Zealand

Tel +642 I 24I 4647

Fax +6493754345

Email a.bartlett@auckland.ac.nz 
Currently, there is no consensus guideline in Australasia or internationally as to the type or degree of donor hepatic steatosis considered safe or acceptable in LT. Appraisal of the degree of hepatic steatosis is highly variable and subjective. ${ }^{2}$ Given the stakes involved - PNF of the transplanted liver in grafts that exceed an acceptable degree of steatosis at one extreme and potential inappropriate discard of an organ at the other - accurate appraisal of the steatotic donor liver is paramount. While an increasing body of literature supports the use of formal evaluation of the donor organ for steatosis prior to LT, the translation of this into clinical practice appears to have been at best ad hoc. ${ }^{2}$

The aim of this survey was to evaluate the current clinical practice amongst liver transplant surgeons in Australasia with regards to the appraisal and use of steatotic donor livers in LT, and to discuss this in the context of the current available evidence.

\section{Methods}

An anonymous online twelve-question survey was emailed to all practicing LT surgeons in Australia and New Zealand $(n=23)$ in January 2010, along with two reminder emails to initial nonresponders. The survey sought to approximate the incidence of steatosis in deceased donor organs retrieved as perceived by LT surgeons, and to determine current practice amongst surgeons from different centers in Australasia in their appraisal and use of steatotic grafts. As most centers do not have standardized guidelines on the evaluation of steatotic donor grafts, individual practices were not assumed to be the same within a center, and therefore surgeons from the same transplant center were invited to participate. At the time this survey was carried out, all deceased-donor livers in New Zealand and the overwhelming majority of deceased-donor livers in Australia were from donation after brain-stem death.

Questions are outlined in Table 1 of the results section. This survey was based on a similar survey conducted in the United Kingdom and United States by Imber et al in 2002, with the permission of the original authors. ${ }^{2}$ As this survey encompassed an audit-based activity, ethics exemption was granted by the Northern Regional Ethics Committee, New Zealand. Results are presented as percentage response rates to each question and rounded to the nearest decimal place.

\section{Results}

The response rate was 83\% (19/23), which encompassed surgeons from all transplant centers and states in Australia and New Zealand, and was therefore considered representative. Some variation in responses by surgeons within a single center was noted, and this occurred across all participating centers. Responses are summarized in Table 1.

\section{Prevalence of steatosis in donor livers in Australasia}

The estimated prevalence of steatosis in donor livers is displayed in Table 1. Over half of the respondents estimated that $40 \%-60 \%$ of the livers retrieved had evidence of steatosis.

\section{Appraising the donor liver}

Ninety percent $(n=17)$ of respondents said they would reject a donor liver as being too fatty based on visual and palpation appraisal at the time of retrieval without seeking histological confirmation. The majority $(n=16 ; 84 \%)$ reported they would use visual and palpation appraisal as the sole means by which to reject a fatty liver when the liver appeared to have severe steatosis $(>60 \%)$. One respondent reported using this method when the liver appeared to have moderate or severe steatosis, and one respondent reported that under no circumstances would they use visual and palpation appraisal alone as grounds to reject a fatty liver.

Respondents were then asked under what circumstances they would seek the histological appraisal of a donor liver. Thirty-two percent $(n=6)$ reported they would do so if the liver "looked bad"; $68 \%(n=13)$ reported they would do so if the liver looked bad and there were other risk factors for steatosis. No respondents routinely used histological appraisal when appraising an organ for use in transplantation, and no respondents used histological appraisal when there were risk factors for steatosis but the liver was felt to be acceptable based on visual and palpation appraisal at retrieval.

If histological appraisal was sought, the majority of respondents would take one or two biopsies (58\% and $32 \%$ respectively), usually at the time of retrieval (79\%). Biopsies were taken from the same spot in the liver each time in the majority of cases (79\%). One respondent reported taking biopsies from the part of the liver that appeared to contain the most steatosis.

Respondents were asked which staining technique they used for evaluation of fatty change in a donor-liver biopsy. Fifty-three percent $(n=10)$ reported using hematoxylin and eosin $(H \& E)$ staining, $16 \%(n=3)$ reported using both $H \& E$ and Oil Red O, 26\% $(n=5)$ were unsure, and one respondent used Oil Red O only.

Respondents were asked whether they used imaging with computed tomography (CT), magnetic resonance imaging, or ultrasound scanning to assess fatty change in a donor liver prior to retrieval. Seventy-four percent $(n=14)$ of respondents never did, and 26\% $(n=5)$ sometimes did. 
Table I Appraisal of hepatic steatosis in liver transplantation: Australasian survey of current practice responses

Can you estimate the percentage of livers you retrieve that show evidence of steatosis?

\begin{tabular}{llllll}
$\mathbf{0 - 2 0} \%$ steatosis & $\mathbf{2 0} \% \mathbf{- 4 0} \%$ steatosis & $\mathbf{4 0} \% \mathbf{- 6 0} \%$ steatosis & $\mathbf{6 0} \% \mathbf{- 8 0} \%$ steatosis & $\mathbf{8 0} \%+$ steatosis & Unsure \\
\hline $5.3 \%$ & $36.8 \%$ & $52.6 \%$ & $0 \%$ & $0 \%$ & $5.3 \%$
\end{tabular}

Do you ever reject a liver as being too fatty using only visual and palpation appraisal, without seeking additional confirmation by histology?

Yes

$89.5 \%$

No

Under what circumstances do you seek the histological appraisal of a donor liver?

\begin{tabular}{llll} 
If it looks bad & $\begin{array}{l}\text { If there are risk factors for } \\
\text { steatosis }\end{array}$ & $\begin{array}{l}\text { If it both looks bad and there are risk } \\
\text { factors for steatosis }\end{array}$ & Never \\
\hline $31.6 \%$ & $0 \%$ & $68.4 \%$ & $0 \%$
\end{tabular}

Under what circumstances do you use only visual and palpation appraisal as the grounds on which to reject a fatty liver?

\begin{tabular}{|c|c|c|c|}
\hline $\begin{array}{l}\text { Liver appears to have } \\
\text { severe }(>60 \%) \text { steatosis }\end{array}$ & $\begin{array}{l}\text { Liver appears to have } \\
\text { moderate }(30 \%-60 \%) \text { or } \\
\text { severe }(>60 \%) \text { steatosis }\end{array}$ & $\begin{array}{l}\text { Suspicion that the liver has fatty } \\
\text { change (mild, moderate or severe) }\end{array}$ & $\begin{array}{l}\text { Under no circumstances do I use } \\
\text { only visual and palpation appraisal } \\
\text { to reject a fatty liver }\end{array}$ \\
\hline $88.9 \%$ & $5.3 \%$ & $0 \%$ & $5.3 \%$ \\
\hline \multicolumn{4}{|c|}{ How many biopsies do you take of the donor liver? } \\
\hline Zero & One & Three & Four \\
\hline $0 \%$ & $57.9 \%$ & $31.6 \%$ & $0 \%$ \\
\hline
\end{tabular}

When do you take a biopsy of the donor liver?

At the time of retrieval On the back bench

$78.9 \% \quad 0 \%$

Either at retrieval or on the back bench I never take a biopsy

Where do you take the donor liver biopsy/biopsies?

From the part of the liver From the same spot

that appears to contain in the liver each time

the most fat

\begin{tabular}{llll}
\hline $5.3 \%$ & $78.9 \%$ & $0 \%$
\end{tabular}

What staining technique is used for evaluation of steatosis in a donor liver at your centre?

\begin{tabular}{lllllll} 
H\&E & Oil Red O & Sudan III & TBS & H\&E and Oil Red O & Unsure & None \\
\hline $52.6 \%$ & $5.3 \%$ & $0 \%$ & $0 \%$ & $15.8 \%$ & $26.3 \%$ & $0 \%$
\end{tabular}

Do you use imaging modalities (CT/MRI/USS) to assess steatosis in a donor liver prior to retrieval?

\begin{tabular}{lll} 
Never & Sometimes & Always \\
\hline $73.7 \%$ & $26.3 \%$ & $0 \%$
\end{tabular}

Do you consider microvesicular fat a risk factor for primary nonfunction (PNF)?

\begin{tabular}{lll} 
Yes & No & Unsure \\
\hline $10.5 \%$ & $78.9 \%$ & $10.5 \%$
\end{tabular}

Above what level of steatosis would you always reject a donor liver, irrespective of other variables?

\begin{tabular}{lllllllll}
$>\mathbf{2 0} \%$ & $>\mathbf{3 0} \%$ & $>\mathbf{4 0} \%$ & $>\mathbf{5 0} \%$ & $>\mathbf{6 0} \%$ & $\mathbf{3 0 0}$ & No limit & Not applicable \\
\hline $0 \%$ & $0 \%$ & $5.3 \%$ & $26.3 \%$ & $52.6 \%$ & $5.3 \%$ & $0 \%$ & $10.5 \%$ & \\
\hline
\end{tabular}

Abbreviations: H\&E, hematoxylin and eosin; CT, computed tomography; MRI, magnetic resonance imaging; USS, ultrasound scanning.

\section{Microvesicular and macrovesicular steatosis}

Respondents were asked whether they considered microvesicular fat a risk factor for the development of PNF in the recipient. Seventy-nine percent did not consider it a risk factor, but $21 \%$ were either unsure or felt it was a risk factor for PNF.

\section{Rejection threshold}

Respondents were asked above what level of steatosis they would always reject a donor liver, irrespective of other variables. The average threshold for rejection of a donor liver in these circumstances was $>60 \%$ steatosis (range $40 \%-80 \%$ ). All data are summarised in Table 1.

\section{Discussion}

Nonalcoholic fatty liver disease is the most commonly cited reason for rejecting a liver at the time of retrieval for transplantation. ${ }^{13}$ In parallel with the burgeoning epidemics of obesity and type II diabetes in Australasia and around the developed world, the prevalence of hepatic steatosis, both in the general population and in those coming forward for organ donation, is predicted to substantially increase in the coming years. ${ }^{14}$ 
This survey of surgeon experience suggests that already a significant proportion of donor livers retrieved in Australasia appear to have steatosis. Half of all respondents estimated the percentage of organs they retrieved that showed evidence of steatosis to be at $40 \%-60 \%$. While the exact prevalence of steatosis in the general population is not well defined, the estimated percentage of steatotic livers encountered by transplant surgeons in this survey is substantially higher than those published in the previous decade, which range from $13 \%$ to $37 \%$, depending on the appraisal tool used. 5,9 While this survey relied on self-reporting of retrospective estimates of steatosis encountered by an individual surgeon, a rising prevalence of donor steatosis is not surprising. The past decade has seen a dramatic increase in rates of obesity, diabetes, and metabolic syndrome in Australian ${ }^{15}$ and New Zealand ${ }^{16}$ adults, conditions which are strongly associated with development of hepatic steatosis. ${ }^{17}$

Several controversies exist as to the type and degree of steatosis that adversely impacts outcome after transplantation. Lipid accumulation within hepatocytes has traditionally been classified morphologically as macrovesicular or microvesicular, based on the presence of either a single large vacuole of lipid displacing the hepatocyte's nucleus (macrovesicular steatosis) or multiple tiny lipid vesicles that accumulate in the hepatocyte's cytoplasm and do not displace the nucleus (microvesicular steatosis). ${ }^{18}$ Obesity, diabetes, and alcohol consumption are the predominant etiologies that result in the development of macrovesicular steatosis, whereas metabolic disorders and toxins are more often attributed as the causative agents for microvesicular steatosis. The severity of steatosis is classified according to the percentage of hepatocytes involved, ${ }^{19}$ with livers being classified as having mild steatosis $(<30 \%$ hepatocytes involved), moderate steatosis ( $30 \%-60 \%)$, or severe steatosis $(>60 \%)$. This simple classification system has been widely adopted in the literature and clinical practice.

Most of the earliest studies evaluating the association between donor steatosis and graft dysfunction in the recipient focused on macrovesicular steatosis. ${ }^{3,20}$ Progressive deterioration in graft function and survival was observed with progression from mild to severe steatosis, with PNF being reported in $87 \%$ of patients that received livers with severe steatosis. ${ }^{5}$ On this basis, it has become widely accepted that livers with severe steatosis $(>60 \%)$ should not be transplanted. However, PNF was reported in up to $13 \%$ of livers with moderate steatosis $(30 \%-60 \%)^{6}$ and was still increased in mild steatosis compared to nonsteatotic livers. ${ }^{5}$ A number of studies over the past decade have been published further evaluating the association between mild and moderate steatosis with poor graft function, and report widely differing outcomes. ${ }^{9-11,21}$ These include an Australian study that reported no difference in PNF rates between moderately steatotic and nonsteatotic livers. ${ }^{9}$

The generally accepted view is that mild macrovesicular steatosis is acceptable and moderate macrovesicular steatosis confers risk of inferior graft function and survival, and therefore these organs should be considered as "marginal" or "extended criteria," and severely (macro)steatotic organs should be routinely discarded. This represents clinical consensus rather than the findings of any meta-analyses, which are currently absent. In keeping with this, the majority (53\%) of respondents in this survey rejected donor livers with $>60 \%$ macrovesicular steatosis. This is similar to the reported practice in the United Kingdom. ${ }^{2}$ Just over a quarter of respondents in this survey took a slightly more conservative approach and used $50 \%$ as their cutoff for rejecting a steatotic liver, which is more in line with American practice. ${ }^{2}$

One area where there appears to be an agreement of evidence is in the setting of microvesicular steatosis, which has consistently been shown not to be associated with graft dysfunction, regardless of the percentage of hepatocyte involvement. ${ }^{22-24}$ Despite this evidence, $11 \%$ of survey respondents still believed microvesicular steatosis was associated with the development of PNF, while a further $11 \%$ were unsure. Microvesicular steatosis has been estimated to be present in $9 \%-25 \%$ of livers retrieved for LT. ${ }^{25}$ Since microvesicular steatosis can only be differentiated from macrovesicular steatosis by histology, then this would suggest that all steatotic livers being considered for outright rejection at retrieval should have the type of steatosis characterized histologically before deciding against their use for LT.

The classification system for severity of steatosis based on the percentage of involved hepatocytes is relatively simple; however, the actual appraisal of the percentage of liver involved by the transplant team during retrieval of an organ is fraught with difficulty and a lack of consistency. Donor risk factors for steatosis, pretransplant imaging using $\mathrm{CT}$ and magnetic resonance imaging, visual and palpation appraisal of a liver by the retrieval team, and liver biopsy have all been used for assessment of donor organs for steatosis. In a previous international survey of practicing US and UK transplant surgeons, subjective appraisal of the donor organ by the transplant team using visual and palpation grounds alone was the predominant means by which a team decided to accept or reject a liver, making organ appraisal one of the least scientific aspects of the transplantation process. ${ }^{2}$ In our survey, $90 \%$ of 
respondents reported that they were prepared to reject a liver as being too steatotic based on visual and palpation grounds alone, without seeking further histological confirmation of the degree of steatosis. The majority ( $89 \%$ ) reported doing this when the liver looked or felt like it had severe steatosis.

A third of the respondents sought further histological appraisal of a liver donor if the liver "looked bad" (but not severely steatotic), and $68 \%$ reported they would do so if the liver "looked bad" and if there were also risk factors for steatosis. None of the respondents routinely used histological appraisal when appraising an organ for use in LT. Interestingly, even if there were risk factors present for steatosis and if the liver was considered acceptable on visual and palpation grounds alone, then no further histological confirmation was sought by any of the respondents. This raises the question of how accurate visual and palpation appraisal is in comparison to other more objective tools, such as biopsy, imaging, and validated risk scores. Steatotic livers are attributed as having a characteristic yellow hue, rounded edges, greasy firm texture, and large size. However, the value of visual and palpation appraisal in accurately quantifying steatosis is variable and highly dependent on the experience of the surgeon. It is most predictive in the setting of severe steatosis where the positive predictive value has been estimated at $71 \%$, but decreases substantially to $17 \%$ in the setting of mild steatosis. Of importance, in the setting of moderate steatosis, where decisions are made around use as a marginal or extended-criteria donor, the positive predictive value of visual and palpation appraisal is only $46 \%{ }^{26}$

Rey et al in 2009 evaluated the disparity between surgical and histological appraisal of steatosis in 36 donor livers deemed unsuitable for LT. Histological assessment was undertaken on a wedge biopsy from each segment of the donor liver and classified according to published criteria analyzing steatosis, inflammation, fibrosis, and liver-cell damage. This study demonstrated that while macroscopic surgical evaluation of the donor liver was important for identifying liver tumors, progressive fibrosis/cirrhosis, vascular variations, and hepatic vein thrombosis, the surgical evaluation of steatosis and early degrees of fibrosis was of low predictive value. Yellow color changes were found to be particularly misleading, and it was possible to observe mild, moderate, and severe steatosis histologically in livers with indistinguishable yellow coloring. Of concern, 16.7\% of the livers discarded on the basis of visual and palpation appraisal as being nontransplantable were within the acceptable histological criteria for transplantation. ${ }^{27}$ In another single-center experience, institution of protocol biopsies of all donor livers resulted in a reduction of PNF rates from
$8.4 \%$ to $1.4 \%$, due to better selection and rejection of organs that fell in the moderate-steatosis category. ${ }^{28}$

Histological appraisal of the donor organ, while more objective, is not always straightforward, owing to regional variation in steatosis and differences in staining techniques. As steatosis does not always uniformly affect a graft, a single biopsy at the time of retrieval may misrepresent the extent of steatosis in the liver. The optimal number of biopsy sites has previously been identified as at least two biopsies from two different segments. ${ }^{29}$ This approach accounted for $95 \%$ of variations in macrosteatosis and microsteatosis. In our survey, only $42 \%$ of respondents took two or more biopsies when seeking histological appraisal of an organ. Controversy also exists as to the most sensitive staining technique to detect fatty infiltration within the hepatocyte. Due to time constraints, frozen sections are more likely to be performed, a system that favors H\&E staining techniques, which are faster and less dependent on technical expertise than such stains as the more sensitive Oil Red $\mathrm{O}$, which is superior for staining lipids. This is reflected in the results of our survey, where H\&E was the predominant stain used.

Imaging assessment of donor steatosis prior to organ retrieval potentially provides a noninvasive means of objectively assessing the whole organ. Nonenhanced CT scanning can accurately predict moderate-to-severe steatosis, but is costly and associated with high false-negative rates in mild steatosis $(<30 \%) .{ }^{30}$ Only $26 \%$ of respondents used imaging modalities to assess for steatosis in donors, and this was on an ad hoc basis. This finding is in line with international practice, and reflects the cost and lack of diagnostic sensitivity of currently available imaging modalities in cadaveric donation.

In 2002, Imber et al conducted a similar survey of LT surgeons in both the UK and the US. Surgeons in both countries reported lower estimates of the percentage of livers retrieved that showed evidence of steatosis than that reported by Australasian surgeons almost a decade later (mean percentage range was $20 \%-40 \%$ in both the UK and the US compared to $40 \%-60 \%$ in our survey). Opinions and practice when evaluating donor livers for steatosis was also found to be diverse in the 2002 survey. Notably, US surgeons tended to adopt a more conservative approach with regards to steatosis assessment than their UK counterparts, utilizing both histological and imaging modalities more frequently, and accepting lower percentage levels of steatosis for LT. In 2002, 50\% of surgeons in the UK never sought histological appraisal of a donor liver, even if the liver looked bad and there were risk factors for steatosis, whereas no US surgeons took this approach. ${ }^{2}$ While objective appraisal of the donor liver in our survey appears 
suboptimal, it has improved comparative to figures originally reported by UK surgeons at the turn of the century.

In conclusion, this survey highlights the significant variation in the appraisal and use of steatotic grafts in Australasia. With rising rates of obesity, the number of donor organs with steatosis is likely to increase further and poses a real threat to the donor pool. It has become clear over the past decade that accurate evaluation and judicious use of normal and donor organs with steatosis is required to optimize the transplant outcomes and ensure maximal use of this scarce resource. Despite this, our survey highlights that there appears to be significant variation between surgeons and centers in the appraisal and use of steatotic grafts, and a divergence of practice with established evidence in some areas. Heavy reliance on macroscopic appraisal by the surgeon of the donor liver was seen in this survey, despite evidence that this has low predictive value in all but the fattiest of livers, potentially resulting in a number of livers that would be considered histologically acceptable being discarded. While histological appraisal of the donor organ is not straightforward, it remains the best means currently available for accurate appraisal of hepatic steatosis. Histological assessment of hepatic steatosis is currently underutilized as a technique by many surgeons in Australasia. As all surgeons in this survey participate in a single common organ-sharing network throughout Australasia, development of best-practice guidelines regarding the appraisal and use of organs procured through this network would seem an appropriate next step. This is likely to remain a challenge given the ongoing controversy within the transplant literature, as well as the absence of a highly sensitive, well-validated method for accurately appraising steatotic livers at the time of donation.

\section{Acknowledgment}

This project was funded by the Auckland District Health Board (ADHB) Charitable Trust.

\section{Disclosure}

The authors report no conflicts of interest in this work.

\section{References}

1. Mokdad AH, Ford ES, Bowman BA, et al. Prevelance of obesity, diabetes and obesity-related health risk factors. JAMA. 2003;289(1):76-79.

2. Imber CJ, St Peter SD, Lopez I, Guiver L, Friend PJ. Current practice regarding the use of fatty livers: a trans-Atlantic survey. Liver Transpl. 2002;8(6):545-549

3. Todo S, Demetris AJ, Makowka L, et al. Primary nonfunction of hepatic allografts with preexisting fatty liver infiltration. Transplantation. 1989;47(5):903-905.

4. Strasberg SM, Howard TK, Molmenti EP, Hertl M. Selecting the donor liver: risk factors for poor function after orthotopic liver transplantation. Hepatology. 1994;20(4 Pt 1):829-838.
5. D'Alessandro AM, Kalayoglu M, Sollinger HW, et al. The predictive value of donor liver biopsies on the development of primary nonfunction after orthotopic liver transplantation. Transplant Proc. 1991;23(1 Pt 2): $1536-1537$.

6. Adam R, Reynes M, Johann M, et al. The outcome of steatotic grafts in liver transplantation. Transplant Proc. 1991;23(1 Pt 2):1538-1540.

7. Busuttil RW, Tanaka K. The utility of marginal donors in liver transplantation. Liver Transpl. 2003;9(7):651-663.

8. Noujaim HM, de Ville de Goyet J, Montero EF, et al. Expanding postmortem donor pool using steatotic liver grafts: a new look. Transplantation. 2009;87(6):919-925.

9. Verran D, Kusyk T, Painter D, et al. Clinical experience gained from the use of 120 steatotic donor livers for orthotopic liver transplantation. Liver Transpl. 2003;9(5):500-505.

10. Chui AK, Haghighi K, Painter D, et al. Donor fatty (steatotic) liver allografts on orthotopic liver transplantation. Transplantation Proc. 1998;30(7):3286-3289.

11. Canelo R, Braun F, Sattler B, et al. Is a fatty liver dangerous for transplantation? Transplant Proc. 1999;31(1-2):414-415.

12. Cameron AM, Ghobrial RM, Yersiz H, et al. Optimal utilisation of donor grafts with extended critera: a single-center experience in over 1000 liver transplants. Ann Surg. 2006;243(6):748-755.

13. Loinaz C, González EM. Marginal donors in liver transplantation. Hepatogastroenterology. 2000;47(31):256-263.

14. Farrell GC, Larter CZ. Nonalcoholic fatty liver disease: from steatosis to cirrhosis. Hepatology. 2006;43(2):S99-S112.

15. Cameron AJ, Welborn TA, Zimmet PZ, et al. Overweight and obesity in Australia: the 1999-2000 Australian diabetes, obesity and lifestyle study (AusDiab). Med J Aust. 2003;178(9):427-432.

16. Gerritsen S, Stefanogiannis N, Galloway Y. A Portrait of Health: Key Results of the 2006/07 New Zealand Health Survey. Wellington: Ministry of Health; 2008.

17. Charlton M. Nonalcoholic fatty liver disease: a review of current understanding and future impact. Clin Gastroenterol Hepatol. 2004;2(12): $1048-1058$

18. Burt AD, Mutton A, Day CP. Diagnosis and interpretation of steatosis and steatohepatitis. Semin Diagn Pathol. 1998;15(4):246-258.

19. Ploeg RJ, D'Alessandro AM, Knechtle SJ, et al. Risk factors for primary dysfunction after liver transplantation - a multivariate analysis. Transplantation. 1993;55(4):807-813.

20. Portmann B, Wight D. Pathology of liver transplantation (excluding rejection). In: Calne R, editor. Liver Transplantation, 2nd ed. London: Grune and Strutton; 1987:437-470.

21. Hayashi M, Fujii K, Kiuchi T, et al. Effects of fatty infiltration of the graft on the outcome of living-related liver transplantation. Transplant Proc. 1999;31(1-2):403.

22. Fishbein TM, Fiel MI, Emre S, et al. Use of livers with microvesicular fat safely expands the donor pool. Transplantation. 1997;64(2): $248-251$.

23. Ureña MA, Ruiz-Delgado FC, González EM, et al. Assessing risk of the use of livers with macro and microsteatosis in a liver transplant program. Transplant Proc. 1998;30(7):3288-3291.

24. Turgeon NA, Sollinger HW, Fernandez LA, et al. Hepatic steatosis and liver transplantation. Curr Opin Organ Transplant. 2004;9(2): 123-129.

25. Garcia Ureña MA, Colina Ruiz-Delgado F, Moreno González E, et al. Hepatic steatosis in liver transplant donors: common feature of donor population? World J Surg. 1998;22(8):837-844.

26. Adam R, Bismuth H, Diamond T, et al. Effect of extended cold ischaemia with UW solution on graft function after liver transplantation. Lancet. 1992;340(8832):1373-1376.

27. Rey JW, Wirges U, Dienes HP, Fries JW. Hepatic steatosis in organ donors: disparity between surgery and histology? Transplant Proc. 2009;41(6):2557-2560.

28. Markin RS, Wisecarver JL, Radio SJ, et al. Frozen section evaluation of donor livers before transplantation. Transplantation. 1993;56(6): 1403-1409. 
29. Frankel WL, Tranovich JG, Salter L, Bumgardner G, Baker P. The optimal number of donor biopsy sites to evaluate liver histology for transplantation. Liver Transpl. 2002;8(11):1044-1047.
30. Park SH, Kim PN, Kim KW, et al. Macrovesicular hepatic steatosis in living liver donors: use of CT for quantitative and qualitative assessment. Radiology. 2006;239(1):105-112.

\section{Publish your work in this journal}

Transplant Research and Risk Management is an international, peerreviewed open access journal focusing on all aspects of transplantation and risk management to achieve optimal outcomes in the recipient improving survival and quality of life. The journal welcomes submitted papers covering original research, basic science, clinical studies, reviews \& evaluations, guidelines, expert opinion and commentary, case reports and extended reports. The manuscript management system is completely online and includes a very quick and fair peer-review system, which is all easy to use. Visit http://www.dovepress.com/ testimonials.php to read real quotes from published authors.

Submit your manuscript here: http://www.dovepress.com/transplant-research-and-risk-management-journal 